\title{
Simulation of $\mathrm{MeV} /$ atom cluster correlations in matter
}

\author{
J. W. Hartman and T. A. Tombrello \\ Basic and Applied Physics, California Institute of Technology, Pasadena, California 91125
}

\author{
S. Bouneau, S. Della Negra, D. Jacquet, Y. Le Beyec, and M. Pautrat \\ Institut de Physique Nucléaire CNRS-IN2P3, F-91406 Orsay Cedex, France
}

(Received 22 February 2000; published 19 September 2000)

\begin{abstract}
We present an efficient algorithm able to predict the trajectories of individual cluster constituents as they penetrate relatively thick amorphous targets. Our algorithm properly treats both the intracluster Coulomb repulsion and the collisions between cluster constituents and target atoms. We have compared our simulation predictions to experimental measurements of the distribution of lateral exit velocities, and demonstrated that the in-target Coulomb explosion of $2 \mathrm{MeV} /$ atom carbon clusters in carbon foils must be shielded with a screening length of less than $2.5 \AA$. We also present a simple phenomenological model for the suppression of the exit-side charge of ions in clusters which depends on the enhanced ionization potential that an electron near an ion feels due to the ion's charged comoving neighbors. By using our simulation algorithm we have predicted the exit correlations of the cluster constituents and verified that the charge suppression model fits the observed charge suppression of ions in clusters to within the experimental uncertainties.

PACS number(s): 36.40. $-\mathrm{c}, 29.40 .-\mathrm{n}, 07.77 .-\mathrm{n}, 34.70 .+\mathrm{e}$
\end{abstract}

\section{INTRODUCTION}

The desire to understand the wealth of effects occurring during cluster-surface interactions motivates the need for microscopic knowledge of the intracluster atomic correlations as the clusters penetrate the surface and pass through solid material. As the cluster travels through the material, the cluster constituents become ionized and the separation between the cluster constituents increases in response to their mutual Coulomb repulsion and also to collisions between the cluster ions and the target atoms. For very thin targets and fast clusters, e.g., tens of nanometers and millions of electronvolts per atom, exiting cluster ions will have roughly the same configuration and length scale as that of the incident cluster, but for progressively thicker targets the cluster constituents will become farther and farther apart. As a direct result, cluster-surface effects which are enhanced by ion-ion proximity will decrease with the intracluster separation until the individual domains of influence separate and the cluster constituents act independently. Thus, by studying physical effects at the exit side of thin foils, one can learn about the length scales of the mechanisms responsible for the effects.

Experimental measurement of the intracluster correlations is not presently possible, so to determine these correlations we have developed an efficient algorithm able to predict the in-target and exit-side intracluster correlations of fast clusters (MeV/atom) through relatively thick (hundreds of $\mu \mathrm{g} / \mathrm{cm}^{2}$ ) carbon targets. When an almost neutral cluster penetrates a target, the electrons which bind the cluster together are stripped away and the positive cores repel each other through their mutual Coulomb repulsion. At the same time, collisions with target atoms redirect and reduce the ions' initial forward momenta, and electronic stopping slows the ions. Our algorithm separates the forces encountered by the cluster ions into two types: collisions between an $\mathrm{MeV}$ cluster ion and a target atom are sudden and appropriately described in the binary collision approximation, while the intracluster forces are relatively constant, persist for a much longer time, may result from many of the other ions in the cluster, and must be treated by a full molecular-dynamics calculation. It is interesting to note that the intracluster forces themselves are not well known. While the cluster ions should certainly repel one another with a hard core and likely repel each other with a Coulomb explosion on some type of effective charge, it is not clear what the strength of the charge is or how effective the screening of the potential is in the target. One of our goals in this paper is to place limits on the importance and strength of the intracluster interactions and so to refine our ability to predict proper exit correlations with this algorithm.

We will discuss results of a recent experiment performed at IPN which studied the effects of target thickness and cluster velocity and size upon the lateral exit-side velocity distributions and the ionic charges of exit-side cluster constituents [1]. The lateral components of exit-side velocities for incident $\mathrm{C}_{n}$ clusters through thin carbon foils were measured by placing a multi-impact position sensitive detector (MIPSD) $356 \mathrm{~mm}$ behind the exit side of the target. The MIPSD consists of two channel plates of $45 \mathrm{~mm}$ diameter with an "anode" of $16 \times 16$ individual anodes. The individual pixels are $2.54 \mathrm{~mm}$ square and so the resolution of the exit velocity cone is approximately $2.54 / 356=7.13$ milliradians. Comparison of the measured and predicted lateral spread of velocities from $\mathrm{C}_{1}$ incident ions provides a test of the C-C scattering cross section used by our algorithm while a similar comparison for $\mathrm{C}_{n>1}$ provides information about the intracluster forces in-target and the initial shape and internal energy of the clusters.

In the same experiment, two parallel and horizontal deflection plates were placed five millimeters behind the cluster target. The charge of the exiting cluster constituents was determined by applying an electric potential across the plates and measuring the subsequent deflection of the cluster constituents with the MIPSD. A strong charge suppression effect (up to $30 \%$ ) was noted and correlated to increasing cluster size and decreasing target thickness. Several authors have reported that, compared to single ions passing through thin 
foils, the average charge state of the constituents of polyatomic projectiles is lower than the charge of an individual atom [2-7] and enhanced electron-capture models which depend on intracluster separations have been proposed to explain the observations $[2,8]$. We present a phenomenological model for charge suppression that supposes that the enhancement of electron capture by the cluster constituents depends strongly on the additional ionization potential felt by an electron near an individual ion when both are in the vicinity of the charged neighboring ions. With our algorithm we can predict correlations based on the intracluster forces and the initial cluster conditions and quantitatively test the model against the measurements.

\section{DESCRIPTION OF INTEGRATION ALGORITHM}

As a cluster of ions penetrates a target, it transfers energy to the target atoms and electrons and starts secondary collision cascades. However, for fast ions the average nuclear stopping is small and the secondary cascade is weak and rapidly left behind the moving cluster. Thus a great computational advantage can be gained by ignoring the secondary cascade and considering only the initial binary collisions between the cluster ions and the target atoms [9]. In our algorithm we describe the ion-atom collisions in the impulse approximation: ions suffer a momentum loss $\vec{p} \rightarrow \vec{p}-\overrightarrow{\delta p}$ every time they collide with a target atom. The momentum transfer during a binary collision is a function of the center-of-mass energy, the impact parameter, and the interaction potential only. A universal formula for this momentum transfer, dubbed the "Magic Formula," has been developed and documented by Beirsack and Haggmark and is at the core of the widely used binary collision simulator TRIM [9]. In our algorithm we also use this formula to describe nuclear stopping of the cluster ions. As an ion travels through a material of density $\rho=1 / a^{3}$ (units $1 / L^{3}$ ), on average it suffers a collision every time it travels a distance $a$. To determine the magnitude and direction of the impulse received during the collision, a target atom position is randomly chosen to lie in a disk of radius $a / 2$ centered on the initial direction of the ion. This determines the impact parameter of the collision, and the magnitude and direction of the impulse received by the moving ion. Of course, electronic stopping is dominant in the $\mathrm{MeV} /$ atom range and must be included. To introduce the effects of electronic straggling in a rational manner, the electronic stopping is correlated to the overlap of the electrons bound to the binary colliders [9].

As the cluster penetrates the target, its ions suffer binary collisions as described above, but between binary collisions, intracluster forces act upon the cluster ions. The ions are comoving, so even weak forces may be important because they must be integrated over relatively long times. Several or all of the cluster ions may be within interaction range of one another during the cluster transit through the material, therefore a full molecular-dynamics integration of the intracluster forces is required. We use a predictor corrector algorithm to integrate these forces between binary collisions. Position is here labeled $\vec{x}$, velocity $\vec{v}$, acceleration $\vec{a}(\vec{x})$, and time step $t$. At each step of the integration, the error in the ion accelera- tions $\vec{a}(\vec{x}+t \vec{v})-\vec{a}(\vec{x})$ is tested to assure that the rate of error introduction into the velocity is small enough that we can assume that the final uncertainty in the ion trajectory at the exit side of the target is less than 0.1 milliradians. Energy of a $2 \mathrm{MeV} /$ atom cluster is conserved to better than $1 \mathrm{eV} / \AA$ under this testing condition. Initially the algorithm attempts to integrate to the next binary collision in a single step, but if it fails to pass the velocity error test, it successively attempts $2,4,8, \ldots, 2^{n}$ steps until all the intermediate error checks are passed.

The appropriate potential to describe the intracluster forces is itself an open question. Physically, there must be at least a hard-core repulsion between the cluster ions; we have included this as a Molliere potential with

$$
\begin{aligned}
V_{M}(r)= & \frac{Z_{1} Z_{2} e^{2}}{r}\left(0.35 e^{-0.3 r / a_{\mathrm{TF}}}+0.55 e^{-1.2 r / a_{\mathrm{TF}}}\right. \\
& +0.10 e^{\left.-6.0 r / a_{\mathrm{TF}}\right)} \\
a_{\mathrm{TF}}= & 0.8854\left(Z_{1}^{1 / 2}+Z_{2}^{1 / 2}\right)^{-2 / 3} 0.529 \AA .
\end{aligned}
$$

In addition to the hard core, electrons stripped from fast ions leave them with a net positive charge which results in a Coulomb explosion as the clusters penetrate the target. The in-target ion charge is a function of the ion velocity and the electronic structure of the target material and is approximated by us to be the Brandt charge as described in Ref. [9]. (We note that this is not the "effective charge" commonly used to relate the electronic stopping power of heavy ions to that of hydrogen but rather an estimate of the true ionization state of the ion.) Because the charges are in a medium with free electrons, we expect that the Coulomb potential will be shielded, thus we assume

$$
V_{C}(r)=\frac{\left[q_{B}(v) e\right]^{2}}{r} e^{-r / \alpha}
$$

where $q_{B}(v)$ is the Brandt charge mentioned above, and $\alpha$ is the screening length set by the ability of the target electrons to shield the ions from one another. The screening length is likely of order $v_{F} / \omega_{0}$ or $v / \omega_{0}$, where $\omega_{0}$ is the plasma frequency of the target and $v_{F}$ and $v$ are, respectively, the Fermi velocity of the electrons in the target and the velocity of the moving ions [10]. For MeV/atom clusters in carbon targets, both these length scales are of order an angstrom.

In this presentation we do not attempt to model the screening length, but rather attempt to fit it by correlating the simulation predictions to the experimental measurements. Decoupling the ion-ion and ion-atom interactions is a seemingly poorly defined process. The true trajectories of ions interacting with each other and the atoms in the lattice are not the same as those they follow under the algorithm defined above. However, in the fast ion limit the error in trajectory is sufficiently small that the difference is insignificant. Typically, as a cluster of fast ions travels forward by a lattice constant of length, there is only an infinitesimal perturbation to the ion velocities and positions due to the intracluster forces. An important point to be made is that the 
influence of the ion-ion interaction over this time scale is very weak. If we assume that the ion-ion interaction is Coulombic, then the displacement due to ion-ion interactions while the ion travels forward by a single lattice constant is on the order of

$$
\begin{aligned}
\left.\delta r\right|_{a} & \simeq \frac{1}{2} \frac{q_{1} q_{2} e^{2} n}{m r^{2}}(\delta t)^{2}, \quad \delta t \simeq a / v \\
& \simeq 0.004\left(\frac{q_{\text {ion }}}{10}\right)^{2} \frac{\mathrm{MeV}}{E} \frac{n}{10}\left(\frac{a}{r}\right)^{2} \AA
\end{aligned}
$$

where $E$ is the ion kinetic energy and $r$ is the separation between ions in the cluster. Because the separation between ions will be on the order of the lattice constant, we see that for $\mathrm{MeV}$ ions, the Coulomb explosion contributes only a slight displacement $(\sim 0.01 \AA)$ to the trajectory of the ion over each lattice constant traversed. Again, it is only because the ions travel forward many lattice constants while still within interaction range of one another that the Coulomb explosion has a noticeable effect.

Similarly, the error introduced by the impulse approximation to the ion-atom scattering trajectory is small for fast ions as evidenced by the strong forward scattering of $\mathrm{MeV}$ ions in solids. Because the velocity perturbations for all but the hardest collisions are of order $0.1 \%$ of the forward velocity, the deviation of the trajectory from the straight-line path can be no more than the same order.

Given the crude limit presented above on the trajectory errors inherent in our algorithm, one can estimate when it should begin to fail. Demanding that $\delta r$ remains smaller than some fraction of an angstrom suggests that the algorithm should be reliable for the integration of trajectories of $\sim \mathrm{MeV} /$ ion clusters.

\section{PREDICTION OF MeV/ATOM $\mathrm{C}_{N}$ EXIT CORRELATIONS}

The initial shapes of cold $\mathrm{C}_{n}{ }^{+}$clusters have been recently measured and found to be either linear or annular for $n$ $<20$, with all $\mathrm{C}_{5}{ }^{+}$linear, two of three $\mathrm{C}_{8}{ }^{+}$clusters linear, and nine of ten $\mathrm{C}_{10}{ }^{+}$clusters annular [11]. $\mathrm{C}_{3}{ }^{+}$is not reported in this reference, but elsewhere $\mathrm{C}_{3}$ (neutral) is predicted to be linear [12]. The bond lengths between carbon atoms in cold $\mathrm{C}_{n}$ clusters are approximately $1.3 \AA$ and the energy required to remove a carbon atom from the end of a linear cluster is of order $6 \mathrm{eV}$ [12]. Further, the energy required to break a single bond in neutral $\mathrm{C}_{8}$ and $\mathrm{C}_{10}$ rings to form linear structures is calculated to be less than $1 \mathrm{eV}$ [12]. The introduction of internal energy to the clusters is a possibly important effect (as discussed below) but a proper treatment would require more than our present understanding of the electronic structure, production, and acceleration of the $\mathrm{C}_{n}$ clusters.

We have used our algorithm to calculate intracluster correlations between ions in linear and annular $2 \mathrm{MeV} /$ atom $\mathrm{C}_{n=1,3,5,8,10}$ clusters at the exit side of $2.2,2.4,3.4,5.3,10.2$, 15.0 , and $40.0 \mu \mathrm{g} / \mathrm{cm}^{2}$ thick carbon foil targets and we present selected results in Figs. 1 and 2. The correlation functions presented in these figures require some explanation. The mean correlation function is defined to be the mean number of neighboring cluster ions that an ion sees within a sphere of radius $r$ centered on itself. Thus for a $C_{3}$ cluster in the shape of a perfect triangle with bond length $c$, the cluster is symmetric with respect to ion position, and the mean correlation function is

$$
c_{3, \text { triangle }}(r)= \begin{cases}0, & r<c \\ 2, & c>r .\end{cases}
$$

For a linear $\mathrm{C}_{3}$ cluster of the same bond length, the correlation function of the ions at the ends of the cluster is zero for $r<c$, one for $c<r<2 c$, and two for $2 c<r$; while for the ion in the center the correlation function is zero for $r<c$ and two for $c<r$. Thus the mean correlation function is

$$
c_{3, \text { line }}(r)= \begin{cases}0, & r<c \\ 1.33=(2+1+1) / 3, & c<r<2 c \\ 2, & 2 c<r .\end{cases}
$$

Correlation functions presented in Figs. 1 and 2 are averaged over at least 1000 cluster penetration events each. For computational efficiency, each cluster penetration event was integrated through $40 \mu \mathrm{g} / \mathrm{cm}^{2}$ of the target but the positions and velocities of each ion in the cluster as it completed passing through 2.2, 2.4, 3.4, 5.3, 10.2, 15.0, and $40.0 \mu \mathrm{g} / \mathrm{cm}^{2}$ of the target were recorded so that correlations could be calculated later. The results for incident linear and annular clusters have been separated and for each the effect of ranging the strength of intracluster forces from Molliere-core-only to Molliere-core plus long-range Coulomb repulsion using the Brandt charge of the moving ions is indicated in each figure. We can expect that the true correlations lie between those indicated for the full unshielded Coulomb repulsion and for the Molliere-core-only calculations. To indicate the importance of screening, we have also included the results of simulations which assumed Molliere-core plus shielded Coulomb; the screening length in these simulations was $2.5 \AA$. Simple interpretation of the mean correlation function can be performed as follows: the mean distance between cluster constituents is $r$ such that $c(r)=1$ and the greatest distance between any two ions in a typical cluster is $r$ such that $c(r)$ $\simeq n-1$. The presence of steps in the correlation function indicates that the cluster remembers its initial (ordered) configuration and is an indication that the target interaction and intracluster forces have done little to disturb the cluster other than to strip its binding electrons away.

There is a strong configurational dependence on the importance of the in-target Coulomb explosion. Forces on the ions in the middle of a linear cluster are balanced and so the ions do not move (relative to their neighbors) until the ions at the end of the cluster are ejected or until collisions with the target atoms change the configuration of the cluster and break this symmetry. In an annular structure, the ions are repelled from the center of the ring and so all ions are free to move and to release the Coulombic energy immediately upon cluster impact. 

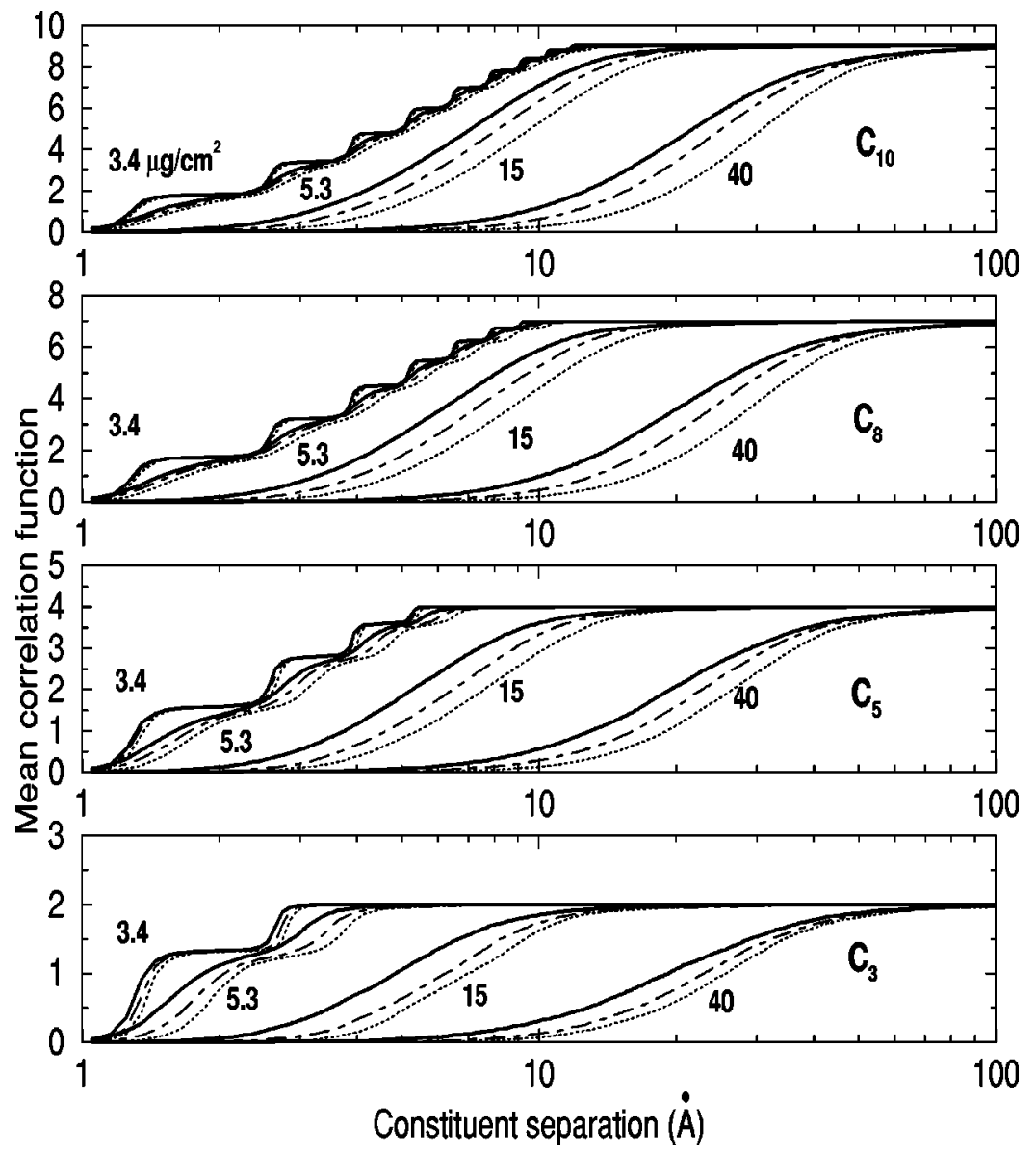

FIG. 1. Intracluster correlation functions for linear $2 \mathrm{MeV}$ /atom $\mathrm{C}_{n}$ clusters after penetration of $3.4,5.3,15.0$, and $40.0 \mu \mathrm{g} / \mathrm{cm}^{2}$ carbon foils. Simulation results are presented for Mollierecore-only interactions (heavy lines), Mollierecore plus shielded-Coulomb (dot-dashed lines), and Molliere-core plus bare Coulomb (dotted lines). The Brandt charge was used for the Coulomb forces, and the screening length of the shielded Coulomb interaction was $2.5 \AA$. Correlation functions for $\mathrm{C}_{n}$ clusters approach $n-1$ at large separation (see text for further details).
Initial internal energy in the clusters may affect the exit correlations of the ions. The $\mathrm{C}_{n}{ }^{+}$clusters used in these experiments were first produced as $\mathrm{C}_{n}{ }^{-}$clusters by sputtering graphite targets, then accelerated to half their final energy in a tandem accelerator, stripped of two electrons by collision with $\mathrm{N}_{2}$ to form $\mathrm{C}_{n}{ }^{+}$, and then accelerated to their final energy. It is reasonable to assume that the final $\mathrm{C}_{n}{ }^{+}$product is in an excited state. We can estimate the maximum effect that internal kinetic energy can have upon the exit correlations by considering the bond strength between the cluster atoms (before impact, obviously). The internal energy of an excited cluster oscillates with time between kinetic and potential energy; at no time during evolution of the excitation modes of the cluster can there be more potential energy stressing a bond than the energy required to break the bond. As noted above, the energy required to remove an atom from $\mathrm{a}_{n}$ chain is of order $6 \mathrm{eV}$, so the maximum internal energy of a cluster should be of order $6 \mathrm{eV}$ times the number of bonds in the cluster ( $n$ for a ring, $n-1$ for a line). This suggests that upon stripping the bonds, each of the atoms should have of order $6 \mathrm{eV}$ kinetic energy relative to the moving cluster. The effect of this energy upon exit-side position correlations is small. A kinetic energy of $6 \mathrm{eV}$ corresponds to a displacement of $t \sqrt{2 \times 6 \mathrm{eV} / m}$, where $m$ is the mass of the atom and $t$ is the transit time in the target. For a cluster of energy $1 \mathrm{MeV} /$ atom with forward velocity $\sqrt{2 \times 1 \mathrm{MeV} / m}$ traveling through a target of thickness $l$ $\left(2.2 \mu \mathrm{g} / \mathrm{cm}^{2} \simeq 100 \AA\right)$, we expect a displacement due to the internal energy of order $l \sqrt{6 \mathrm{eV} / 1 \mathrm{MeV}} \simeq 0.002 l$, i.e., of order $1 \AA$ for a $10 \mu \mathrm{g} / \mathrm{cm}^{2}$ thick carbon foil. For thinner foils the internal velocity displaces the ions even less.

\section{CALCULATION OF ION EXIT ANGLE}

We can both test the nuclear stopping cross section used by our integrator and place limits on the strength of the intarget forces between cluster constituents by studying the distribution of exit velocities of the $\mathrm{C}_{n}$ cluster constituents from the thin carbon foils. The lateral spread of cluster constituent exit angles was measured by projecting the exiting ions onto an MIPSD with $16 \times 162.54 \mathrm{~mm}$ pixels located $356 \mathrm{~mm}$ behind the carbon foils [1]. Angular resolution of the MIPSD in this configuration is $2.54 / 356=7.13$ milliradians. From Figs. 1 and 2 it is clear that the ions exit all but the thickest foils relatively close to each other, so to predict properly the final distribution of exit velocities, we must treat the Coulomb explosion that occurs as the comoving cluster constituents travel from the exit side of the foil to the MIPSD. Using the positions and velocities of the cluster constituents upon exiting each target, a new integration was started using unshielded Coulomb forces on the mean exit charge of the exiting cluster ions. Mean exit charge was measured at the same time as the lateral spot size and was reported in Ref. [1]. For improved numerical precision, integration was performed in the center-of-mass frame of the cluster, and continued until the potential energy remaining in 

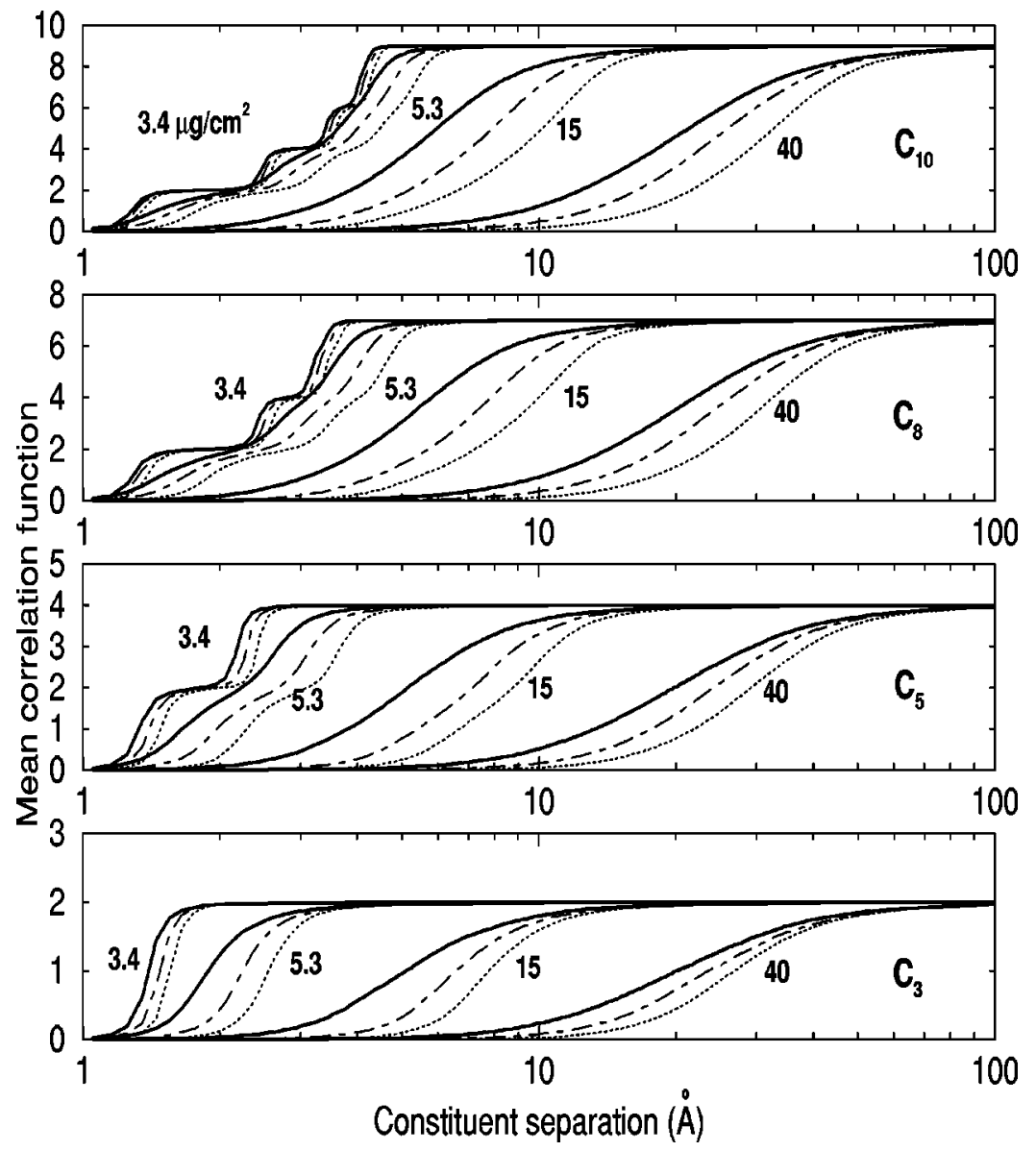

FIG. 2. Intracluster correlation functions for annular $2 \mathrm{MeV}$ /atom $\mathrm{C}_{n}$ clusters after penetration of $3.4,5.3,15.0$, and $40.0 \mu \mathrm{g} / \mathrm{cm}^{2}$ carbon foils. See the text and the caption of Fig. 1 for further details. the spatial configuration of the cluster was $1 \%$ of the kinetic energy. In these simulations, it was found that the intracluster Coulomb explosion was completed by the time the cluster has traveled about one micrometer from the back of the target toward the detector. At the end of the integration, the direction cosines of the ion velocities (after transformation back into the laboratory frame) were recorded for comparison to the experimentally measured impact positions on the MIPSD.

During experimental measurement of the spot projected onto the MIPSD, a potential was applied perpendicular to the direction of the cluster beam to measure the mean ion charge by deflection. This effect was not introduced into the simulations; thus, to compare experiment to simulation we must study the distributions in the direction perpendicular to the applied potential.

In Fig. 3 we present predictions for the final lateral velocity distributions of $2 \mathrm{MeV}$ /atom linear $\mathrm{C}_{n=1,3,5,8}$ clusters after passing through 2.2 and $40 \mu \mathrm{g} / \mathrm{cm}^{2}$ thick carbon foils. We have assumed that there are no long-range Coulomb forces between cluster constituents while they are in the target and that the only intracluster interactions in the target are due to the hard-core repulsions of the ions. The $2 \mathrm{MeV} /$ atom $\mathrm{C}_{n}$ clusters traversing the $2.2 \mu \mathrm{g} / \mathrm{cm}^{2}$ target are merely stripped of their bonds by the target, so the final lateral velocity distributions of clusters exiting this target are primarily due to the Coulomb explosion behind the target, and are strongly dependent on cluster size. For thicker targets, collisions with target atoms spread the atoms apart, so that upon exit there is less energy available for the behind-target Coulomb explosion. In this case the dependence of the distribution of lateral velocities upon cluster size is weakened; note that the distributions of lateral velocities behind the $40 \mu \mathrm{g} / \mathrm{cm}^{2}$ target are independent of cluster size.

Comparison of simulation results to experimental spectra obtained by the MIPSD requires consideration of the measurement process. Because the angular resolution of the detector is 7.13 milliradians and the widths of the spectra we wish to measure are often of the same order or smaller, there may be a strong "binning" effect for the narrowest spectra. For example, imagine that the entire distribution of impact parameters on the MIPSD lies within a single pixel. Then, the width of the distribution, as calculated by the root mean square (RMS) of the lateral pixel number, is zero. If the MIPSD is shifted so that the peak of the distribution lies on the boundary between two pixels, then the distribution is split evenly between two pixels and the RMS lateral pixel number is one-half. During acquisition of experimental data, care was taken to center the distributions on a single pixel, and so when comparing to simulation predictions we have chosen the same condition for the simulations. Another important effect that must be considered is detector "saturation" during highly correlated multiple ion impacts on the same pixel of the MIPSD. Because the detector recovery time is much longer than the coincidence time of multiple ion impacts from the same cluster event, discrimination be- 


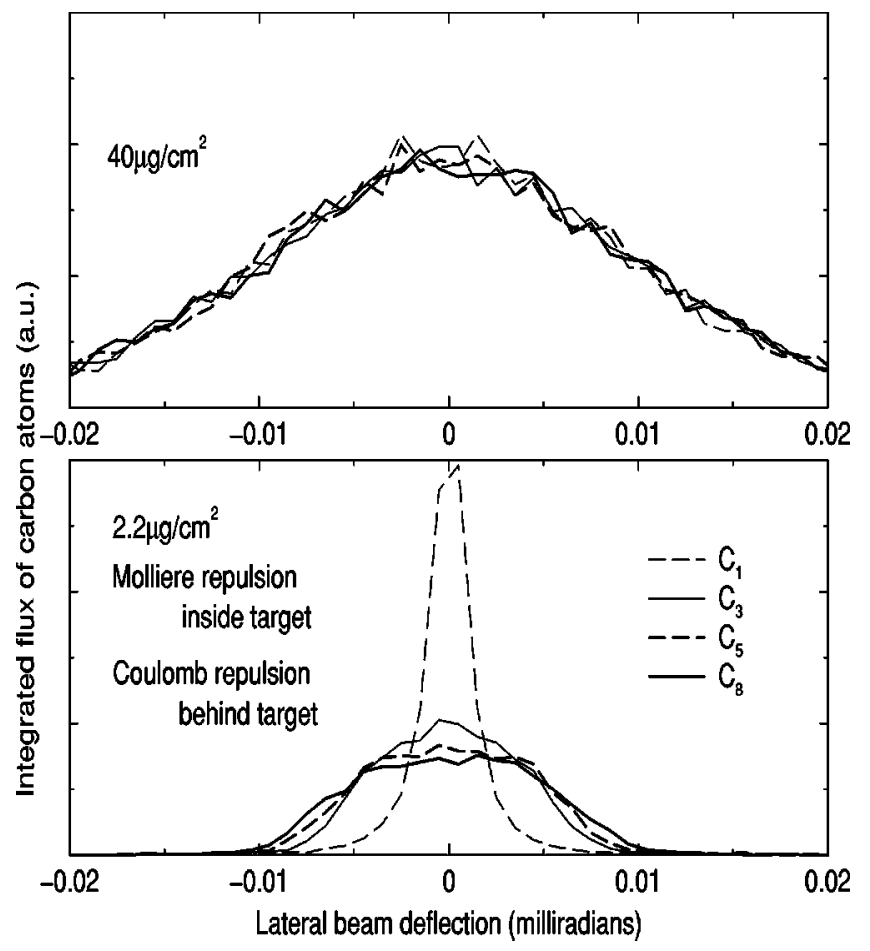

FIG. 3. Lateral velocity distributions for $2 \mathrm{MeV} /$ atom cluster constituents after passing through thin carbon foils and after the Coulomb explosion behind the target has exhausted. Lateral velocity is expressed as a ratio of the forward exit velocity, and so the distribution represents the "spot" that would be projected onto a detector normal to the incident beam of clusters.

tween single ion impacts and these coincident multiple ion impacts on a single pixel of the MIPSD requires amplitude discrimination of the detection pulse. Amplitude discrimination was not performed in these experiments and this effect must be accounted for in the simulated distributions before a comparison can be made.

The single ion detection efficiency was measured to be $55 \%$. If $m$ ions from the same cluster event strike a single pixel, the probability that $l$ of them start electron cascades is thus

$$
\left(\begin{array}{c}
m \\
l
\end{array}\right) \epsilon^{l}(1-\epsilon)^{m-l}, \quad \epsilon=0.55
$$

For $l=0$ no count is measured while for $l=1, \ldots, m$ one count is measured, so the probability for the detection of an $m$-ion impact on the pixel is

$$
\begin{aligned}
f & =0\left(\begin{array}{c}
m \\
0
\end{array}\right) \epsilon^{0}(1-\epsilon)^{m-0}+\sum_{l=1, m} 1\left(\begin{array}{c}
m \\
l
\end{array}\right) \epsilon^{l}(1-\epsilon)^{m-l} \\
& =1-(1-\epsilon)^{m} .
\end{aligned}
$$

Thus if a simulated cluster event results in $m$ ions striking a single pixel, the number of "counts" in the pixel increases not by $m$ but rather by $1-(1-\epsilon)^{m}$. Before comparison of

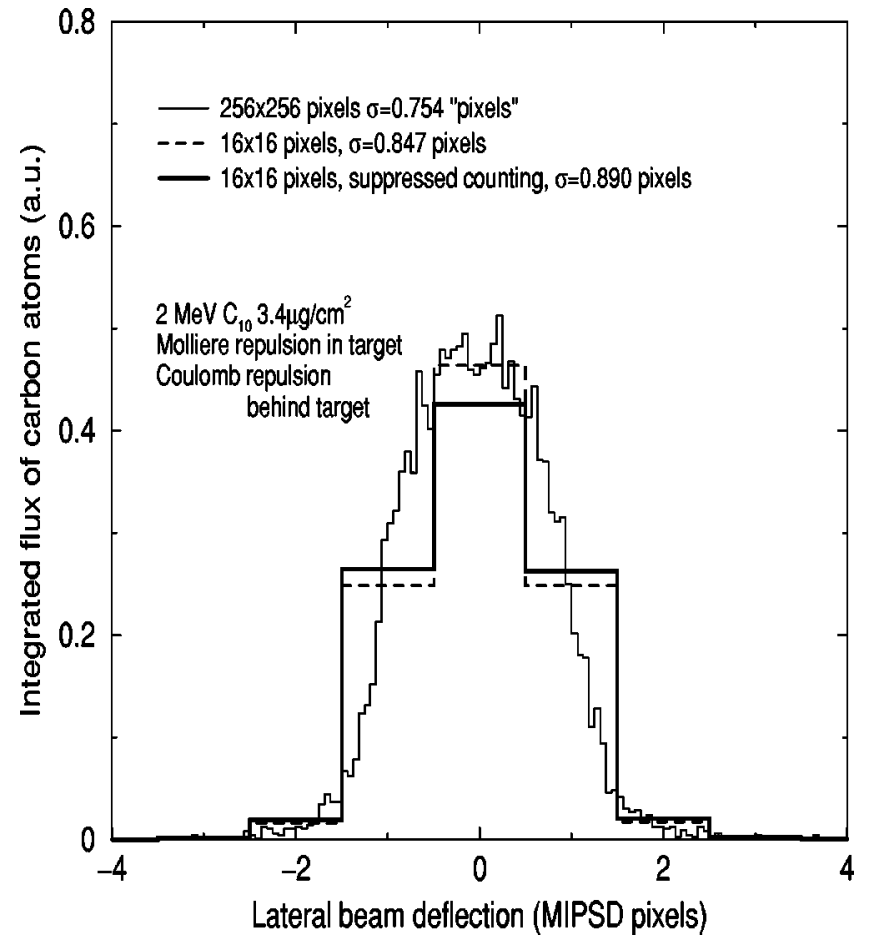

FIG. 4. Schematic of physical effects that must be introduced to the simulated lateral velocity distribution before comparison to the MIPSD-observed distribution can be made.

the simulated results to the measured value in the pixel, this correction factor must be introduced to the simulations on a cluster-by-cluster basis.

In Fig. 4 we have presented a predicted distribution binned first with $256 \times 256$ and then with $16 \times 16$ resolution. To demonstrate the effect of discretization on the apparent width of the distribution, we have calculated the RMS spot size for both resolutions in terms of the $16 \times 16$ pixels: poorer resolution leads to broader apparent widths. Also, in the same figure, we have included the effect of suppressed counting that is suffered by the MIPSD when operated without amplitude discrimination. This effect lowers the counts in the most heavily impacted pixels, further broadening the apparent distribution.

Once these measurement effects are introduced to the simulated distributions, we find satisfactory agreement between measured and predicted lateral velocity distributions. In Fig. 5 we present a comparison of predicted and measured lateral velocity distribution widths for 1,2 , and $4 \mathrm{MeV} /$ atom $\mathrm{C}_{1}$ and $\mathrm{C}_{5}$ clusters behind carbon foils of thickness up to $40 \mu \mathrm{g} / \mathrm{cm}^{2}$. And in Fig. 6 we present a similar comparison of spot size for $2 \mathrm{MeV} /$ atom $\mathrm{C}_{n=1,3,5,8,10}$ clusters. Results are presented in terms of detector pixels; a pixel was approximately seven milliradians from edge to edge in the geometry of the experiment (see Table I). Statistical uncertainty in the predicted widths was calculated by assuming that the uncertainty of the number of counts in each bin was described by Poisson statistics. Then the variance in each bin could be assumed to be $N p(1-p)$, where $N$ is the number of counts in the bin, $N_{\text {tot }}$ is the total number of atomic fragments from the ensemble of $\mathrm{C}_{n}$ decompositions at the given energy and 


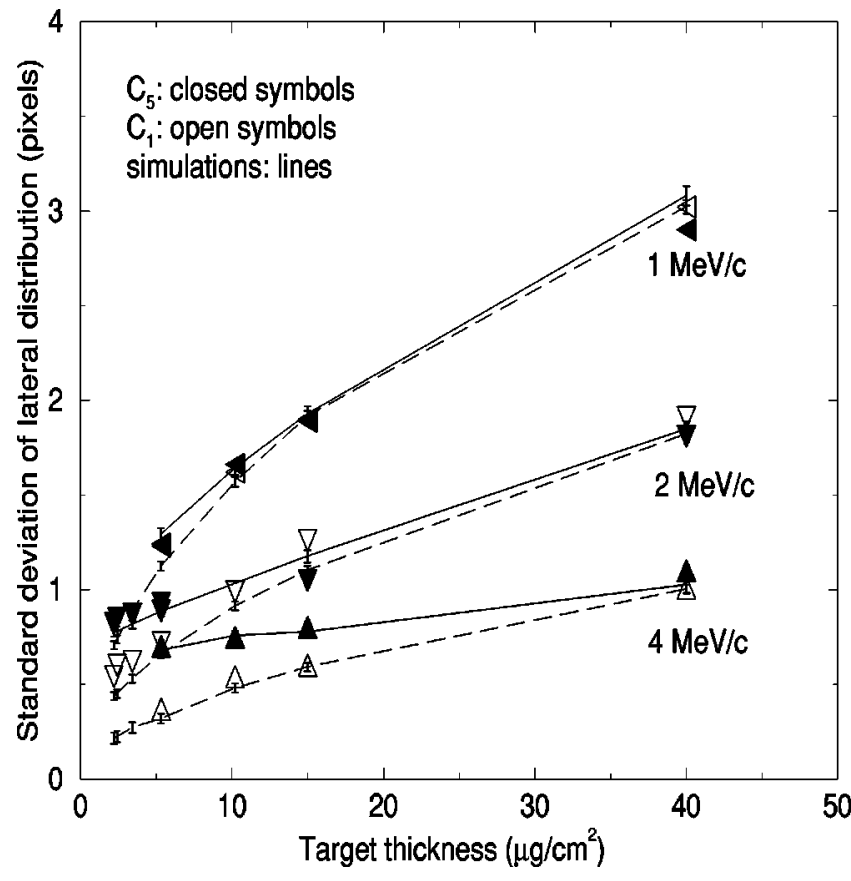

FIG. 5. Comparison of predicted and measured exit-side velocity distributions from carbon foil thicknesses of up to $40 \mu \mathrm{g} / \mathrm{cm}^{2}$ for $\mathrm{C}_{1}$ and $\mathrm{C}_{5}$ clusters of incident energy 1,2 , and $4 \mathrm{MeV} /$ atom. To avoid visual clutter, only the uncertainties in the predictions are included; see Table I for uncertainty in the measurements.

target thickness, and $p=N / N_{\text {tot }}$ is taken as an estimate for the intrinsic probability that an atomic fragment from the $\mathrm{C}_{n}$ cluster strikes the bin. Given these estimates for the variance of the number of cluster fragments striking each bin, standard propagation-of-error techniques were used to estimate the uncertainty in the prediction widths.

Even with the introduction of measurement effects to the simulated distributions, the predicted distributions tend to be slightly narrower than the observed distributions; this is particularly true for the $2 \mathrm{MeV} /$ atom observations. If we assume that the $\mathrm{C}-\mathrm{C}$ scattering cross section is too weak, and scale the range of the interaction potential in the Magic Formula up by to $10 \%$, we find better agreement between prediction and observation. Alternatively, we can assume that the measured target thicknesses or the assumed electronic stopping powers were $10 \%$ too low, or that the incident beam had of order one milliradians of divergence, or that all of the above effects contribute in smaller amounts. Because there are so many parameters we can adjust to fit the experimental data better, we have chosen to adjust none, and present predictions based on the "book" values for nuclear and electronic stopping. While the offset between measurement and prediction must remain unexplained until more discriminating experiments are designed and performed, the trends in the measured and simulated distributions are clear and are easy to compare.

In Fig. 7 we present the measured and predicted distribution widths as a function of cluster size for $2 \mathrm{MeV} /$ atom clusters behind the $40 \mu \mathrm{g} / \mathrm{cm}^{2}$ target. The experimentally measured $\mathrm{C}_{5}$ distribution width is 1.81 pixels but was measured under different detection conditions than the $\mathrm{C}_{1}, \mathrm{C}_{3}$,

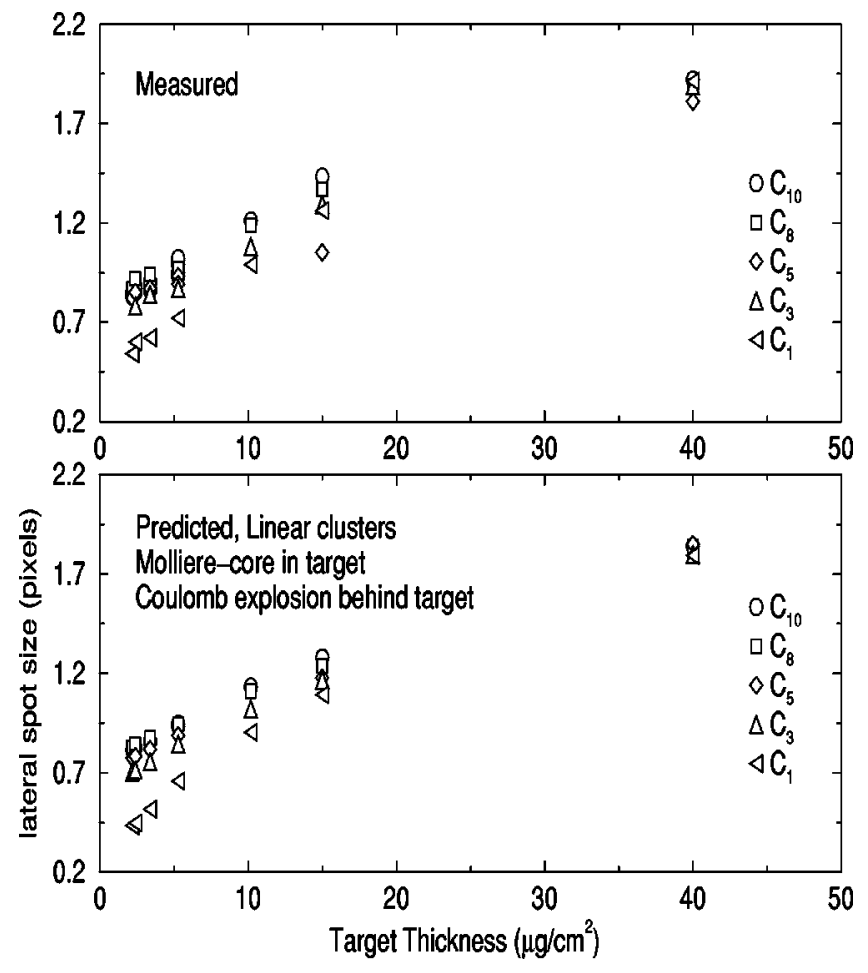

FIG. 6. Comparison of predicted and measured exit side velocity distributions from carbon foil thicknesses of up to $40 \mu \mathrm{g} / \mathrm{cm}^{2}$ for $\mathrm{C}_{1}, \mathrm{C}_{3}, \mathrm{C}_{5}, \mathrm{C}_{8}$, and $\mathrm{C}_{10}$ clusters of incident energy $2 \mathrm{MeV} /$ atom. Statistical uncertainty in the predictions is smaller than the symbols and uncertainty in the measurements is indicated in Table I.

$\mathrm{C}_{8}$, and $\mathrm{C}_{10}$ and so is not displayed. All simulations included a repulsive Molliere potential which provided a hard core interaction between ions. A screened in-target intracluster Coulomb repulsion was also included to study its effect on final lateral velocity distributions. In these sets of simulations, the screening length was chosen to be either $\infty$ (no screening), $5.0 \AA, 2.5 \AA$, or 0 (Molliere core only). A clear trend is seen: for all the simulations with an in-target intracluster force greater than the Molliere repulsion, the distribution widths are seen to increase with cluster size. As seen in Fig. 7, a strong dependence of the distribution width on cluster size contrasts sharply with the experimental observation. Thus, we conclude that the Coulomb repulsion of the 2 $\mathrm{MeV} /$ atom $\mathrm{C}_{n}$ cluster constituents in the carbon target must be shielded with a screening length of less than $2.5 \AA$.

Demonstration that the screening length of Coulombic forces between $2 \mathrm{MeV} /$ atom carbon ions in carbon is less than $2.5 \AA$ is of fundamental interest. In the most primitive estimate of shielding, one expects the screening length to be of order $v / \omega_{0}$, where $\omega_{0}$ is the plasma frequency of the carbon target and $v$ is either ion velocity or the Fermi velocity. For slow ions, $\left(v_{\text {ion }}<v_{\text {Fermi }}\right)$, electrons can respond to the ions' positions and arrange themselves so as to shield the ions from each other, but the sharpness of the screening is limited by the kinetic energy (the Fermi energy) of the electrons in the target. For very fast ions $\left(v_{\text {ion }} \gg v_{\text {Fermi }}\right)$ electronic shielding is less effective because the electrons' inertia prevent them from responding to the passing ions before the 
TABLE I. Measured spot sizes of 1, 2, and $4 \mathrm{MeV} /$ atom $\mathrm{C}_{n}$ clusters seen at the MPISD after passing through carbon foils of thickness noted. One pixel corresponds to approximately seven milliradians of angular spread.

\begin{tabular}{|c|c|c|c|c|c|c|c|}
\hline Cluster & $\begin{array}{c}E_{\text {beam }} \\
(\mathrm{MeV})\end{array}$ & $\begin{array}{l}\text { Target thickness } \\
\qquad\left(\mu \mathrm{g} / \mathrm{cm}^{2}\right)\end{array}$ & $\begin{array}{l}\text { Sigma } \\
\text { (pixels) }\end{array}$ & Cluster & $\begin{array}{c}E_{\text {beam }} \\
(\mathrm{MeV})\end{array}$ & $\begin{array}{l}\text { Target thickness } \\
\qquad\left(\mu \mathrm{g} / \mathrm{cm}^{2}\right)\end{array}$ & $\begin{array}{l}\text { Sigma } \\
\text { (pixels) }\end{array}$ \\
\hline \multirow[t]{9}{*}{ C10 } & 1.0 & 2.20 & $1.17 \pm 0.07$ & \multirow{9}{*}{$\mathrm{C} 3$} & 1.0 & 5.30 & $1.24 \pm 0.05$ \\
\hline & 1.0 & 5.30 & $1.40 \pm 0.07$ & & 1.0 & 10.20 & $1.66 \pm 0.06$ \\
\hline & 2.0 & 2.20 & $0.84 \pm 0.05$ & & 1.0 & 15.00 & $1.89 \pm 0.08$ \\
\hline & 2.0 & 2.40 & $0.86 \pm 0.05$ & & 1.0 & 40.00 & $2.90 \pm 0.10$ \\
\hline & 2.0 & 3.40 & $0.88 \pm 0.05$ & & 2.0 & 2.40 & $0.78 \pm 0.05$ \\
\hline & 2.0 & 5.30 & $0.95 \pm 0.05$ & & 2.0 & 3.40 & $0.84 \pm 0.05$ \\
\hline & 2.0 & 10.20 & $1.21 \pm 0.06$ & & 2.0 & 5.30 & $0.87 \pm 0.05$ \\
\hline & 2.0 & 15.00 & $1.43 \pm 0.07$ & & 2.0 & 10.20 & $1.08 \pm 0.05$ \\
\hline & 2.0 & 40.00 & $1.92 \pm 0.09$ & & 2.0 & 15.00 & $1.29 \pm 0.05$ \\
\hline \multirow[t]{7}{*}{$\mathrm{C} 8$} & 2.0 & 2.20 & $0.87 \pm 0.05$ & \multirow{17}{*}{$\mathrm{C} 1$} & 2.0 & 40.00 & $1.89 \pm 0.08$ \\
\hline & 2.0 & 2.40 & $0.92 \pm 0.05$ & & 3.8 & 5.30 & $0.35 \pm 0.15$ \\
\hline & 2.0 & 3.40 & $0.94 \pm 0.05$ & & 3.8 & 10.20 & $0.5 \pm 0.05$ \\
\hline & 2.0 & 5.30 & $0.97 \pm 0.05$ & & 3.8 & 15.00 & $0.6 \pm 0.05$ \\
\hline & 2.0 & 10.20 & $1.19 \pm 0.05$ & & 3.8 & 40.00 & $1.0 \pm 0.05$ \\
\hline & 2.0 & 15.00 & $1.37 \pm 0.06$ & & 1.9 & 2.20 & $0.54 \pm 0.10$ \\
\hline & 2.0 & 40.00 & $1.89 \pm 0.08$ & & 1.9 & 2.40 & $0.60 \pm 0.10$ \\
\hline \multirow[t]{10}{*}{$\mathrm{C} 5$} & 4.0 & 5.30 & $0.70 \pm 0.05$ & & 1.9 & 3.40 & $0.62 \pm 0.10$ \\
\hline & 4.0 & 10.20 & $0.75 \pm 0.05$ & & 1.9 & 5.30 & $0.72 \pm 0.05$ \\
\hline & 4.0 & 15.00 & $0.80 \pm 0.05$ & & 1.9 & 10.20 & $1.0 \pm 0.05$ \\
\hline & 4.0 & 40.00 & $1.10 \pm 0.06$ & & 1.9 & 15.00 & $1.26 \pm 0.05$ \\
\hline & 2.0 & 5.30 & $0.9 \pm 0.05$ & & 1.9 & 40.00 & $1.91 \pm 0.08$ \\
\hline & 2.0 & 15.00 & $1.09 \pm 0.05$ & & 1.0 & 5.30 & $1.23 \pm 0.07$ \\
\hline & 2.0 & 40.00 & $1.74 \pm 0.07$ & & 1.0 & 10.20 & $1.62 \pm 0.08$ \\
\hline & 2.0 & 2.20 & $0.82 \pm 0.05$ & & 1.0 & 15.00 & $1.90 \pm 0.08$ \\
\hline & 2.0 & 2.40 & $0.85 \pm 0.05$ & & 1.0 & 40.00 & $3.02 \pm 0.12$ \\
\hline & 2.0 & 3.40 & $0.87 \pm 0.05$ & & & & \\
\hline
\end{tabular}

ions travel out of range, here we expect the shielding length to be of order $v_{\text {ion }} / \omega_{0}$. Thus the screening length is of order the greater of $v_{\text {ion }} / \omega_{0}$ and $v_{\text {Fermi }} / \omega_{0}$. The mean unit volume of a carbon atom in the target is approximately $(2.068 \AA)^{3}$; if we assume that there are four conduction electrons per target ion, then the free-electron density, in atomic units, is $n_{e}=4(0.529 \AA / 2.068 \AA)^{3} / a_{0}^{3}=0.067 / a_{0}^{3} \quad\left(a_{0}=0.529 \AA\right)$. Thus the Fermi velocity is $v_{F}=\left(3 \pi^{2} n_{e}\right)^{1 / 3}=1.26 v_{0} \quad\left(v_{0}\right.$ $=22 \AA / \mathrm{fs})$, and the plasma frequency is $\omega_{0}=\left(4 \pi n_{e}\right)^{1 / 2}$ $=0.917 v_{0} / a_{0}$. A $2 \mathrm{MeV}$ carbon ion has a velocity of $56 \AA / f s \simeq 2.5 v_{0}$ (about twice the Fermi velocity) and so we expect the screening length to be of order $2.5 v_{0} /\left(0.917 v_{0} / a_{0}\right)=1.5 \AA$. We note that this is compatible with the maximum screening length required above to fit our simulation results to the experimental observations.

\section{RELATION OF CHARGE SUPPRESSION TO INTRACLUSTER CORRELATIONS}

The effect of target thickness and cluster size upon charge of the carbon ions exiting the back of carbon targets up to $40 \mu \mathrm{g} / \mathrm{cm}^{2}$ thick was measured for $1 \mathrm{MeV} /$ atom $\mathrm{C}_{n=1,5,10}, 2$ MeV/atom $\mathrm{C}_{n=1,3,5,8,10}$, and $4 \mathrm{MeV} /$ atom $\mathrm{C}_{1,5}$ clusters and recently published in Ref. [1]. A strong charge suppression effect (up to $30 \%$ ) was noted for the clusters $(n>1)$ and correlated to increasing cluster size and decreasing target thickness. Simple models for charge suppression can be constructed by supposing that the effect is due to enhanced electron capture by the cluster which depends on the additional ionization potential required for an electron to escape the ion when both are in the vicinity of the neighboring charged ions. If we assume that the charge suppression effect is entirely due to this additional ionization potential felt by an electron on an ion due to the proximity of the ion's comoving charged neighbors, then to linear order in the change in potential we can write

$$
q_{i}=q_{0}(v)-\beta \Delta I_{i}
$$

where $q_{i}$ is the ion's charge, $q_{0}(v)$ is the equilibrium charge of an independent ion traveling at the ion's velocity,

$$
\Delta I_{i}=\sum_{j(\neq i)} q_{j}\left(\frac{e^{-r_{i j} / \alpha}}{r_{i j}}\right)
$$

is the additional ionization energy required to remove each electron from the proximity of the ion and away from the cluster, and $\beta$ is a constant which may depend on ion veloc- 


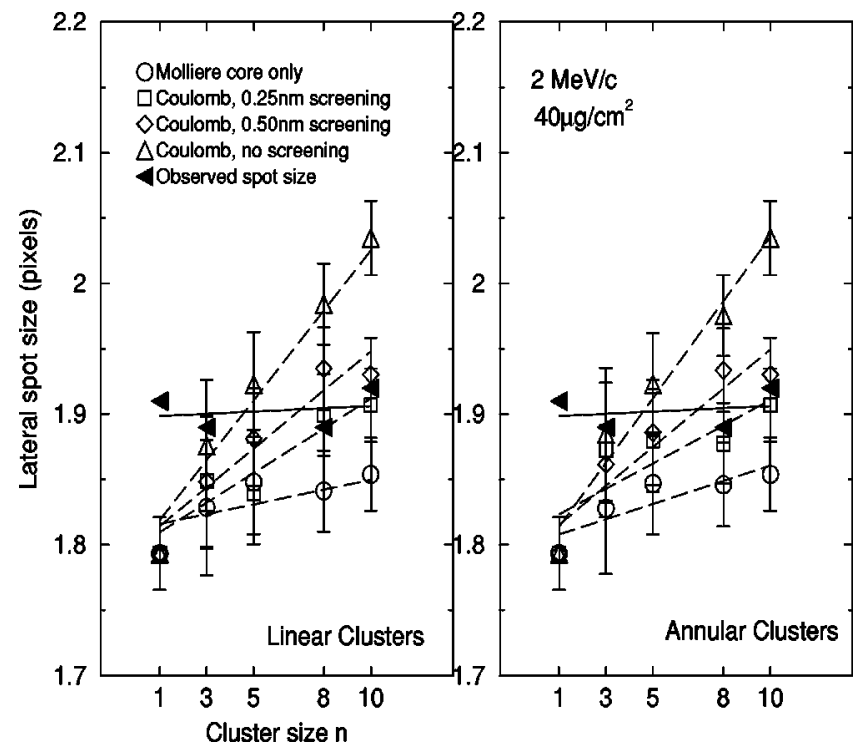

FIG. 7. The widths of the lateral velocity distributions predicted by simulations which include an in-target Coulomb explosion show a clear dependence upon cluster size which is not observed in the experimentally observed distributions. See Table I for the uncertainties in the measured spot sizes.

ity, target material, etc. Here $\alpha$ is a shielding length introduced by the screening effects of electrons between the cluster constituents. Inside the target $\alpha$ may be very small. As found previously, the characteristic length scale for screening of the Coulombic potential for $2 \mathrm{MeV}$ carbon ions in a carbon target must be less than $2.5 \AA$. Upon exit, the electrons of the target are left behind and screening length increases to infinity. For calculation of the additional ionization potential felt by an electron upon cluster exit, the limit $\alpha \rightarrow \infty$ is appropriate: if $\alpha$ is small, there must still be many electrons which may be captured by the ions in order to reduce their charge. As the electrons are captured by the individual ions or escape from the cluster, $\alpha \rightarrow \infty$. Thus the relevant depth of the potential is that from which the last free electrons must escape.

We can evaluate the supposition that cluster ion charge suppression is a simple function of enhanced ionization potential at the exit side of the target by using the correlations predicted by our algorithm. We note that introducing Eq. (9) into Eq. (8) forms a closed set of $n$ linear equations that can be solved for the $q_{i}$ in terms of the correlations $r_{i j}$. However, for simplicity, we evaluate the suppression model in terms of the mean-field approximation: $q_{i} \rightarrow \bar{q}$ and

$$
\Delta I_{i} \rightarrow \frac{1}{n} \sum_{i} \sum_{j \neq i} \frac{\bar{q}}{r_{i j}} \equiv \bar{q} \chi .
$$

Note that the suppression is self-limiting: $\Delta I$ is proportional to $\bar{q}$ but Eq. (8) requires that $\bar{q}$ decrease when $\Delta I$ increases. For this reason we have separated the geometrical contribution to $\Delta I$ and labeled it $\chi$. Then we can solve for the ratio of the reduced charge to the independent charge as a function of the geometrical contribution to the ionization potential

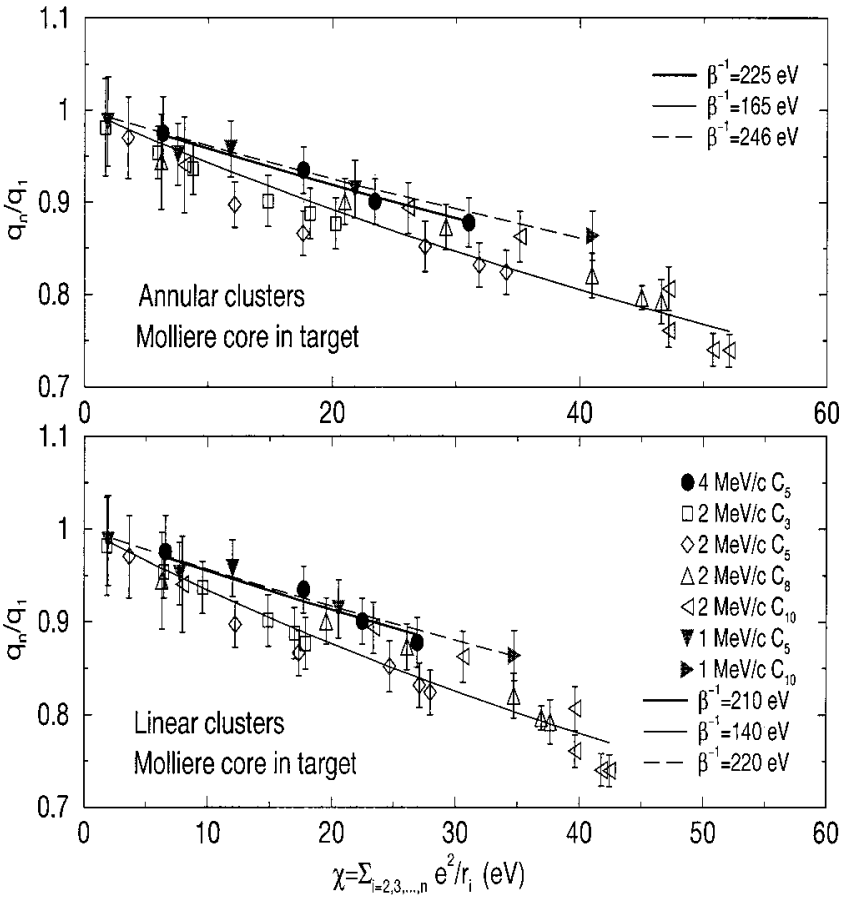

FIG. 8. Ratio of observed cluster-ion to single-ion exit charge as a function of the geometric contribution to the enhanced ionization potential felt by electrons near a cluster ion. Lines represent fits to the predicted dependence after the data sets have been separated by incident energy. See text for further details.

$$
\frac{\bar{q}}{q_{0}(v)}=\frac{1}{1+\beta \chi} .
$$

Equation (11) suggests that the ratio of the mean exit charge for ions in a cluster to the mean exit charge of an independent ion of the same velocity should be a universal function of the geometrical contribution to the ionization potential, $\chi$. In Fig. 8 we plot the experimentally observed mean exit ratios versus the simulation predicted geometrical potential for both incident lines and rings. The in-target interaction potential was assumed to be Molliere core, and no internal kinetic energy was included. Included in the plot are all the data for which measurements of both the independent ion charge and the mean cluster ion charge were made.

If we assume that the clusters are linear, then the constant $\beta$ in Eq. (11) is approximately $1 / 150 \mathrm{eV}$. We note that if we fit the $1 \mathrm{MeV} /$ atom, $2 \mathrm{MeV} /$ atom, and $4 \mathrm{MeV} /$ atom data separately, $1 / \beta_{1 \mathrm{MeV}}=220 \mathrm{eV}, 1 / \beta_{2 \mathrm{MeV}}=140 \mathrm{eV}$, and $1 / \beta_{4 \mathrm{MeV}}=210 \mathrm{eV}$, suggesting that $\beta$ may indeed depend weakly on exit energy. If instead we assume that the clusters are annular, we find $1 / \beta_{1 \mathrm{MeV}}=246 \mathrm{eV}, 1 / \beta_{2 \mathrm{MeV}}=165 \mathrm{eV}$, and $1 / \beta_{4 \mathrm{MeV}}=225 \mathrm{eV}$, but the data still collapse to a universal curve. Thus to within the error bars of the experimental measurements we find that the suppression of ion charge due to the proximity of nearby comoving charged neighbors can be written as a simple function of the additional ionization energy required to remove an electron from the proximity of the cluster. 
The values of $\beta$ required to fit the ionization model to the experimental measurements are, in fact, not dependent on the in-target intracluster forces used to calculate the exit positions of the cluster ions. This is because, for thin targets, the simulation results demonstrate that the in-target repulsion does not contribute significantly to the exit configuration of the clusters (see Figs. 1 and 2). In Fig. 8 the rightmost point of a given cluster size and energy corresponds to the thinnest target, and the universal nature of the charge suppression model can be confirmed independently of the interaction potential by considering only those points in the figure. Then the fact that the data points for thicker targets also lie on the same curve can be taken to indicate that the in-target cluster explosion has been modeled correctly, e.g., that the forces we have chosen to model the in-target ion-ion repulsion are correct.

It is tantalizing to note that $1 / \beta$ is of order the electronic stopping power times the expected screening length of intracluster interactions $\left(d E / d x \sim 150 \mathrm{eV} / \AA\right.$ and $\alpha \sim v / \omega_{0}$ $\sim \sqrt{E / 2 \mathrm{MeV}} 1.5 \AA$ ). Because both electronic stopping and ionization are related to the inability of electrons to follow adiabatically developing orbitals, it would be gratifying to be able to write the suppression effect in terms of the electronic stopping. However, while the magnitude is correct, the trend is not. The stopping powers are $123 \mathrm{eV} / \AA$, $156 \mathrm{eV} / \AA$, and $164 \mathrm{eV} / \AA$ and the screening lengths are of order $1.1 \AA, 1.5$ $\AA$, and $3.0 \AA$ for the $1 \mathrm{MeV}, 2 \mathrm{MeV}$, and $4 \mathrm{MeV}$ carbon ions, respectively. Thus, while $(d E / d x) \alpha$ is monotonically increasing with energy, $1 / \beta$ displays a minimum between 1 $\mathrm{MeV} /$ atom and $4 \mathrm{MeV} /$ atom. To explain this effect one would need to look beyond our simplistic model. We have only attempted to correlate increasing ionization potential to the decreasing ion charge state. This is motivated by the expectation that it is easier to capture free electrons with and harder to lose bound electrons from a deeper potential well. We have not tried to predict the frequencies with which bound electrons attempt to exit and free electrons attempt to enter the bound states.

\section{CONCLUSIONS}

We have presented an efficient algorithm able to describe the microscopic correlations of fast ionic clusters in matter, and used it to calculate correlations between 1, 2, and 4 $\mathrm{MeV} /$ atom $\mathrm{C}_{n}$ clusters as they penetrate thin carbon foils up to $40 \mu \mathrm{g} / \mathrm{cm}^{2}$ thick. For thin targets, we have placed limits on the influence that internal kinetic energy in the clusters can have on the exit correlations, and, by studying how the distribution of lateral exit velocities depends on cluster size and in-target intracluster forces, we have demonstrated that the in-target Coulomb explosion of $2 \mathrm{MeV}$ carbon ions in carbon targets must be shielded with a screening length of less than $2.5 \AA$.

We have used predictions of intracluster correlations to demonstrate that the exit-side charge suppression of cluster ions can be correlated simply to the enhanced ionization potential felt by an electron near an ion due to the ion's comoving charged neighbors. For $\mathrm{C}$ clusters exiting carbon targets with energies of order 1-4 MeV/atom, the strength of the suppression effect is found to depend weakly on exit energy and to display a maximum between $1 \mathrm{MeV} /$ atom and $4 \mathrm{MeV} /$ atom.

By putting strict bonds on the strength of the in-target interaction potential, we increase our ability to predict exitside correlations with our simulation algorithm. However, at present our ability to predict the true exit position correlations is limited by a lack of knowledge of the initial configuration and internal energy of the $\mathrm{C}_{n}$ clusters.

\section{ACKNOWLEDGMENTS}

T.A.T. is supported in part by the National Science Foundation (Grant No. DMR97-30839). The authors gratefully acknowledge G. Maynard for valuable discussions.
[1] A. Brunelle, S. Della-Negra, J. Depauw, D. Jacquet, Y. Le Beyec, and M. Pautrat, Phys. Rev. A 59, 4456 (1999).

[2] D. Maor, P.J. Cooney, A. Faibis, E.P. Kanter, W. Koenig, and B.J. Zabransky, Phys. Rev. A 32, 105 (1985).

[3] A. Faibis, G. Goldring, M. Hass, R. Kaim, I. Plesser, and Z. Vager, Nucl. Instrum. Methods Phys. Res. 194, 299 (1982).

[4] A. Breskin, A. Faibis, G. Goldring, M. Hass, R. Kaim, Z. Vager, and N. Zwang, Phys. Rev. Lett. 42, 369 (1979).

[5] I. Plesser, E.P. Kanter, and Z. Vager, Phys. Rev. A 29, 1103 (1984).

[6] M.J. Gaillard, J.C. Poizat, A. Ratkowski, and J. Rémillieux, Nucl. Instrum. Methods 132, 69 (1976).

[7] B. Mazuy, A. Belkacem, M. Chevalliere, M.J. Gaillard, J.C.
Poizat, and J. Rémillieux, Nucl. Instrum. Methods Phys. Res. B 28, 497 (1987); 33, 105 (1988).

[8] M.F. Steuer and R.H. Ritchie, Nucl. Instrum. Methods Phys. Res. B 40/41, 372 (1989).

[9] J.F. Ziegler, J.P. Beirsack, and U. Littmark, The Stopping and Range of Ions in Solids (Pergamon Press, New York, 1985), and references therein.

[10] W. Brandt, Atomic Collisions in Solids (Plenum, New York, 1975), Vol 1, p. 261.

[11] R. Bouyer, Ph.D. thesis, Universite Paris XI Orsay, 1995 (unpublished).

[12] P. Joyes, Les Agrégats Inorganiques Élémentaires (Les Editions de Physiques, Paris, 1990). 